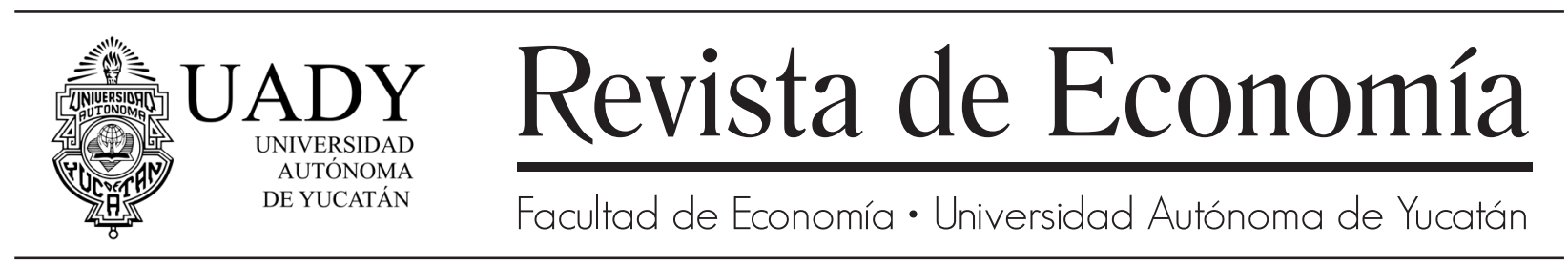

Las metas de inflación y su impacto en la incertidumbre inflacionaria: evidencia empírica para América Latina y el Sudeste Asiático* - Inflation targeting and their impact on inflation uncertainty: empirical evidence for Latin America and Southeast Asia

$$
\text { Eduardo Rosas Rojas }{ }^{1} \text { Juan Carlos Baltazar Escalona² Javier Lapa Guzmán }{ }^{3}
$$

\title{
Resumen
}

Se estudia la relación de retroalimentación entre la inflación y la incertidumbre inflacionaria; además, se estiman los efectos asimétricos que presentan las buenas y malas noticias en la determinación de la varianza condicional de la inflación; así como el impacto que ha tenido la adopción del régimen de metas de inflación (MI) en atenuar la incertidumbre inflacionaria. Para este propósito la investigación se enfoca en dos grupos de economías: i) la región latinoaméricana (Colombia, México, Perú y Uruguay) y la región del Sudeste Asiático (Corea del Sur, Filipinas, Indonesia y Tailandia). La metodología econométrica plantea un esquema $\operatorname{SARIMA}(p, d, q)(P, D, Q) \_S$ GJRGARCH $(p, q)-M$. La evidencia empírica muestra el cumplimiento de la hipótesis de Cukierman y Meltzer para México, Perú, Uruguay, Corea del Sur, Filipinas, Indonesia y Tailandia; en tanto que el cumplimiento de la hipótesis de Friedman y Ball se presenta en Colombia, México, Perú, Uruguay, Filipinas y Tailandia. Finalmente, se identificó que después de la adopción del régimen de MI la persistencia de la incertidumbre inflacionaria ha disminuido en todas las economías analizadas; pero con mejores resultados para las economías asiáticas en los principales agregados macroeconómicos.

Palabras clave: metas de inflación; incertidumbre inflacionaria; modelos de volatilidad condicional autorregresiva.

\footnotetext{
*Esta investigación se realizó con el apoyo del cuerpo académico en economía financiera de la Universidad Autónoma del Estado de México, Centro Universitario UAEM Valle de México.

1- Adscripción: Universidad Autónoma del Estado de México, Valle de México. Correo electrónico: erosasr@uaemex.mx (D) ORCID: https://orcid.org/0000-0002-7255-7778

2- Adscripción: Universidad Autónoma del Estado de México, Valle de México. Correo electrónico: jcbaltazare@uaemex.mx (D) ORCID: https://orcid.org/0000-0002-0478-3036

3- Adscripción: Universidad Autónoma del Estado de México, Valle de México. Correo electrónico: jlapag@uaemex.mx (D) ORCID: https://orcid.org/0000-0001-9302-5319
}

RECEPCIÓN: 28 de abril de 2019 ACEPTACIÓN: 19 de septiembre de 2019 REVISTA DE ECONOMÍA: Vol. 37- Núm 94 ENERO A JUNIO DE 2020: Págs. 81-106 


\section{$\searrow$ Abstract}

The feedback relationship between inflation and inflation uncertainty was studied; in addition, the asymmetric effects of good and bad news in determining the conditional variance of inflation were estimated; as well as the impact that the adoption of Inflation Targeting (IT) regime has had on mitigating inflation uncertainty. For this purpose, the research focused on two groups of economies: i) the Latin American region (Colombia, Mexico, Peru and Uruguay); and the Southeast Asian region (South Korea, the Philippines, Indonesia and Thailand). The econometric methodology proposes a SARIMA scheme SARIMA(p, d, q) (P,D,Q)_S-GJRGARCH(p,q)-M. Empirical evidence shows compliance with the Cukierman and Meltzer hypothesis for Mexico, Peru, Uruguay, South Korea, the Philippines, Indonesia and Thailand; while, compliance with the Friedman and Ball hypothesis is presented in Colombia, Mexico, Peru, Uruguay, the Philippines and Thailand. Finally, it was identified that after the adoption of the IT regime the persistence of inflationary uncertainty has decreased in all the economies analyzed; but with better results for Asian economies in the main macroeconomic aggregates.

Keywords: inflation targeting; inflation uncertainty; autoregressive conditional volatility models.

JEL Classification: C22, C51, E31, D81.

\section{Introducción}

En el actual contexto de globalización financiera, la banca central se ha vuelto mucho más poderosa e importante en comparación con la era de Bretton Woods, en la que dichas instituciones habían tenido un papel bastante pasivo en la economía doméstica. A la par del proceso, la forma en que los bancos centrales operaron su política monetaria cambió drásticamente en las últimas tres décadas. En este paradigma, la transparencia, la independencia y la credibilidad de los bancos centrales han sido características ampliamente destacadas y elogiadas (Frankel, 2010). Es así como en el nuevo esquema, denominado metas de inflación (MI), la política monetaria se ha circunscrito a un objetivo general: lograr la estabilidad de los precios a través de una sola medida, el índice de precios al consumidor.

No obstante, para analizar apropiadamente esta variable, es importante enfocarse no solamente en su nivel esperado; sino también, en su volatilidad. Existe una abundante literatura sobre los diferentes canales a través de los cuales esta incertidumbre distorsiona la toma de decisiones respecto a los ahorros e inversiones futuras, la eficiencia en la asignación de recursos y sobre el nivel de producción real (Holland, 1993). La volatilidad de los precios presenta dos tipos de efectos económicos. El primero, denominado efecto ex ante, se presenta cuando la incertidumbre inflacionaria provoca que, las empresas y los consumidores, tomen decisiones económicas que difieren de aquellas que se habrían realizado en otra circunstancia; es decir, las decisiones anticipan la futura inflación. El segundo efecto toma lugar después de que las decisiones han sido tomadas, 
este efecto ex post ocurre cuando la inflación difiere de lo que se había esperado (Golob, 1994). La mayor parte de la literatura que ha investigado el impacto generado por la implementación del régimen de $\mathrm{Ml}$ se ha centrado en los efectos de esta estrategia monetaria en el nivel y la volatilidad de la inflación, con énfasis en las economías desarrolladas. Sin embargo, aún existen pocos trabajos de investigación de dicho fenómeno en dos de las principales regiones con economías emergentes, América Latina y el Sudeste Asiático. En estas regiones, el esquema de MI se adoptó ante la incapacidad de los regímenes de tipo de cambio fijos para reducir permanentemente las tasas de inflación. La evidencia empírica existente sobre los efectos de la implementación del régimen de $\mathrm{MI}$ aún es controvertida y contradictoria. Por un lado, se enfatiza la debilidad, la incapacidad y las limitaciones de esta estrategia para estabilizar el nivel y la volatilidad de la inflación, y conseguir, simultáneamente, bajas tasas de desempleo y un alto crecimiento económico (Brito y Bystedt, 2010; Carrasco y Ferreiro, 2011). Por otro lado, se ha presentado evidencia favorable sobre el establecimiento del régimen de $\mathrm{Ml}$ en economías en desarrollo, en donde se muestra una disminución en la tasa de inflación y en su incertidumbre (Gonçalves y Salles, 2008; Lin y Ye, 2009; Batini y Laxton 2007).

La presente investigación busca proporcionar evidencia sobre tres temas que siguen siendo debatibles y que presentan diferencias significativas en su concepción. El primer tema es sobre la causalidad existente entre el nivel de la inflación y la incertidumbre inflacionaria, y el signo con que estas se determinan entre sí. El segundo tiene que ver con la adecuada aproximación de la volatilidad de la inflación, y que se ha logrado estimar mediante modelos de heteroscedasticidad condicional autorregresiva generalizada y sus diferentes especificaciones. Finalmente, el tercero hace referencia al impacto que ha tenido la adopción del régimen de metas de inflación (MI) en atenuar la incertidumbre. En la primera muestra se estudian las economías de Colombia, México, Perú y Uruguay; en tanto que, para la segunda, se consideran los países de Corea del Sur, Filipinas, Indonesia y Tailandia. Los datos que se emplean son mensuales, esto permite que se pueda capturar el efecto estacional del fenómeno económico. Además de que se utiliza el mayor número de datos disponibles para cada una de las economías.

Los resultados muestran el cumplimiento de la hipótesis de Cukierman y Meltzer para México, Perú, Uruguay, Corea del Sur, Filipinas, Indonesia y Tailandia; por otro lado, se identifica el cumplimiento de la hipótesis de Friedman y Ball para Colombia, México, Perú, Uruguay, Filipinas y Tailandia; mientras que, la hipótesis de Pourgerami y Maskus se cumple únicamente para Corea del Sur. También se puede identificar la presencia de un efecto asimétrico de los choques positivos de la varianza condicional de la inflación sobre la tasa de cambio porcentual del índice de precios al consumidor para las economías de Colombia, México, Perú e Indonesia. Otro resultado de suma importancia es que, después de la implementación del régimen de MI, la persistencia de la volatilidad ha disminuido en todas las economías analizadas; sin embargo, los resultados en términos de las principales variables reales son considerablemente mejores para las cuatro economías asiáticas.

El trabajo de investigación se encuentra organizado como sigue: la segunda sección muestra una revisión de la literatura sobre las principales características de la relación de retroalimentación entre la inflación y su incertidumbre inflacionaria, sobre las técnicas econométricas que se han utilizado para aproximar su medición al concepto de incertidumbre de Friedman (1977) y sobre las características del régimen de MI implementado por la ortodoxia; en la tercera, se pre- 
senta la metodología econométrica para la construcción de un esquema , y así poder identificar la naturaleza de la inflación y su incertidumbre; en la cuarta sección se desarrolla la estimación y los resultados empíricos; posteriormente, en la quinta sección se presenta la discusión sobre la aplicación del régimen de $\mathrm{MI}$ en las dos regiones analizadas. Finalmente, en la sexta sección se redactan las conclusiones.

\section{Revisión de la literatura}

La tasa de inflación al igual que la incertidumbre inflacionaria, recientemente han atraído, de manera significativa, la atención de los tomadores de decisiones y de la comunidad académica.

Aunque el entendimiento de la interacción del nivel de precios y de su incertidumbre inflacionaria, juega un rol central en la implementación exitosa de la política monetaria; aún no existe un consenso teórico o empírico sobre la naturaleza de esta relación. Fue Friedman (1977), quién informalmente postuló los potenciales efectos adversos del nexo inflación-incertidumbre inflacionaria en la actividad económica real y Ball (1992), quién propuso una justificación matemática para esta última hipótesis, en el contexto de un juego teórico de información asimétrica establecido entre la autoridad monetaria y el público, predijo que una alta inflación podría potencialmente llevar a una mayor incertidumbre inflacionaria en el futuro. A estos postulados se les ha denominado la hipótesis de Friedman-Ball.

En oposición a estas teorías, Pourgerami y Maskus (1987); así como Ungar y Zilberfarb (1993) desarrollaron otro enfoque al constituir un modelo teórico que parte de la suposición de que, los agentes gastan más recursos en pronosticar la inflación cuando aumenta la inflación. De esta forma, defienden la idea de que la inflación en sí misma genera una dinámica que provoca niveles de precios mucho más anticipados y, por lo tanto, disminuye la incertidumbre inflacionaria en el futuro.

Respecto a la dirección de causalidad inversa que podrían presentar estas variables, Cukierman y Meltzer (1986) argumentaron que, un incremento en la incertidumbre inflacionaria, induce a una mayor tasa de inflación promedio ya que el banco central podría asumir una posición oportunista creando sorpresas inflacionarias y, de esta manera, estimular el crecimiento del producto. En contraposición, Holland (1995) sugiere que, si la hipótesis de Friedman es correcta, entonces el banco central tendría incentivos para reducir la inflación promedio cuando la incertidumbre inflacionaria sea alta. El banco central buscaría reducir la oferta monetaria para reducir la inflación; de esta forma postula una relación negativa entre dichas variables. El resumen de las hipótesis descritas se observa en el cuadro 1. 
Cuadro 1. Hipótesis económicas sobre inflación e incertidumbre inflacionaria

\begin{tabular}{|l|l|l|}
\hline \multicolumn{1}{|c|}{ Hipótesis } & \multicolumn{1}{|c|}{ Efecto causal } & \multicolumn{1}{c|}{ Impacto } \\
\hline Friedman(1977) - Ball (1992) & Inflación $\left(\pi_{\mathrm{t}}\right)$ a incertidumbre $\left(h_{\mathrm{t}}\right)$ & Positivo $(+)$ \\
\hline $\begin{array}{l}\text { Pourgerami - Maskus (1987) / Ungar- } \\
\text { Zilberfarb (1993) }\end{array}$ & Inflación $\left(\pi_{\mathrm{t}}\right)$ a incertidumbre $\left(h_{\mathrm{t}}\right)$ & Negativo $(-)$ \\
\hline Cukierman - Meltzer (1986) & Incertidumbre $\left(h_{\mathrm{t}}\right)$ a inflación $\left(\pi_{\mathrm{t}}\right)$ & Positivo $(+)$ \\
\hline Holland (1995) & Incertidumbre $\left(h_{\mathrm{t}}\right)$ a inflación $\left(\pi_{\mathrm{t}}\right)$ & Negativo $(-)$ \\
\hline
\end{tabular}

Fuente: Elaboración propia

En las economías donde se ha implementado el régimen de metas de inflación la autoridad monetaria, generalmente, prefiere disminuir la tasa de crecimiento de la oferta monetaria, con ello busca eliminar el efecto negativo de la incertidumbre inflacionaria que surge de niveles altos de inflación. Para hacer esto, los formuladores de política podrían tener motivos de estabilización a largo plazo o estar regidos por algún mecanismo de compromiso que requiera estabilidad en el nivel de precios. Entre las principales investigaciones desarrolladas para la región latinoamericana y del Sudeste Asiático sobre la relación de causalidad entre la inflación y su incertidumbre se encuentran las consideradas en el cuadro 2.

Cuadro 2. Investigaciones desarrolladas para Latinoamericana y el Sudeste Asiático

\begin{tabular}{|c|c|c|c|c|c|}
\hline & Articulo & Metodología & Periodo & Paises & Relación de causalidad \\
\hline \multirow{7}{*}{ 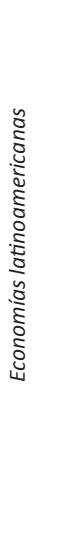 } & \multirow{2}{*}{$\begin{array}{l}\text { Daal, Naka y Sánchez } \\
\text { (2005) }\end{array}$} & \multirow[t]{2}{*}{ PGARCH } & \multirow[t]{2}{*}{$1957-2004$} & \multirow{2}{*}{$\begin{array}{l}4 \text { países emergentes de } \\
\text { Latinoamérica. }\end{array}$} & $\pi_{\mathrm{t}} \rightarrow h_{\mathrm{t}}$ (México) \\
\hline & & & & & $\begin{array}{c}\pi_{\mathrm{t}} \leftrightarrow h_{\mathrm{t}} \text { (Argentina, Co- } \\
\text { lombia y Venezuela) }\end{array}$ \\
\hline & Grier y Grier (2006) & GARCH-M & $1972-2001$ & México & $\pi_{\mathrm{t}} \rightarrow h_{\mathrm{t}}$ \\
\hline & Miles (2009) & GARCH-M & $1980-2007$ & Colombia & $\pi_{\mathrm{t}} \rightarrow h_{\mathrm{t}}$ \\
\hline & Broto (2008) & Q-STARCH & $1995-2006$ & $\begin{array}{l}8 \text { Economías latinoa- } \\
\text { mericanas }\end{array}$ & $\begin{array}{c}\pi_{\mathrm{t}} \leftrightarrow h_{\mathrm{t}} \text { (Argentina, } \\
\text { Basil, Chile, Colombia, } \\
\text { Ecuador, México, Perú y } \\
\text { Uruguay) }\end{array}$ \\
\hline & $\begin{array}{l}\text { Perrotini y Rodríguez } \\
\text { (2012) }\end{array}$ & GARCH-M & 1929-2009 & México & $\pi_{\mathrm{t}} \leftrightarrow h_{\mathrm{t}}$ \\
\hline & $\begin{array}{c}\text { Caballero y Caballero } \\
\text { (2015) }\end{array}$ & SARIMA-GARCH-M & $1990-2013$ & Bolivia & $\pi_{\mathrm{t}} \leftrightarrow h_{\mathrm{t}}$ \\
\hline \multirow{3}{*}{ 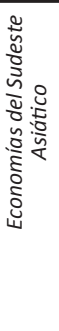 } & Chen, Shen y Xie (2006) & $\begin{array}{l}\text { Modelo de regresión } \\
\text { flexible no lineal }\end{array}$ & $1965-2003$ & $\begin{array}{c}4 \text { países del Sudeste } \\
\text { Asiático }\end{array}$ & $\begin{array}{c}h_{\mathrm{t}} \rightarrow \pi_{\mathrm{t}} \text { (Hong Kong) } \\
\pi_{\mathrm{t}} \leftrightarrow h_{\mathrm{t}} \text { (Taiwán, Singapur } \\
\text { y Corea del Sur) }\end{array}$ \\
\hline & $\begin{array}{l}\text { Bredin, Elder y Fountas } \\
\text { (2009) }\end{array}$ & VAR - GARCH - M & $1963-2005$ & $\begin{array}{l}4 \text { países del Sudeste } \\
\text { Asiático y la India }\end{array}$ & $\begin{array}{c}h_{\mathrm{t}} \rightarrow \pi_{\mathrm{t}} \text { India, Corea del } \\
\text { Sur, Malasia, Filipinas y } \\
\text { Singapur }\end{array}$ \\
\hline & Jiranyakul y Opiela (2010) & AR (p) - EGARCH (1,1)-M & $1970-2007$ & $\begin{array}{c}5 \text { países del Sudeste } \\
\text { Asiático }\end{array}$ & $\begin{array}{c}\pi_{\mathrm{t}} \leftrightarrow h_{\mathrm{t}} \text { (Indonesia, Mala- } \\
\text { sia, Filipinas, Singapur y } \\
\text { Tailandia) }\end{array}$ \\
\hline
\end{tabular}




\begin{tabular}{|l|l|l|l|c|c|}
\hline & Mohd, et al. (2013) & EGARCH & $1980-2011$ & $\begin{array}{c}5 \text { países del Sudeste } \\
\text { Asiático }\end{array}$ & $\begin{array}{c}\pi_{\mathrm{t}} \rightarrow h_{\mathrm{t}} \text { (Malasia, Tailan- } \\
\text { dia, Singapure, Indonesia } \\
\text { y Filipinas) }\end{array}$ \\
\hline
\end{tabular}

Nota: $\pi_{\mathrm{t}}=$ valor esperado de la tasa de cambio porcentual del índice de precios al consumidor (IPC) y $h_{\mathrm{t}}$ es la varianza condicional de la inflación (incertidumbre inflacionaria), $\rightarrow$ relación unidireccional, $\leftrightarrow$ relación bidireccional.

En la época previa al desarrollo de modelos de heteroscedasticidad condicional algunas investigaciones empíricas utilizaban la dispersión de las secciones cruzada sobre encuestas de pronósticos de inflación como medida de la incertidumbre inflacionaria (Evans, 1991). Sin embargo, observar una baja dispersión ex post no implica una baja incertidumbre, ya que los agentes económicos aún podrían tener un poco de información sobre la inflación y, por tanto, considerar el futuro como altamente incierto. A partir del documento seminal de Engle (1982) la metodología de heteroscedasticidad condicional autorregresiva (ARCH) y la ampliación generealizada (GARCH) de Bollerslev (1986) estos modelos han emergido como el enfoque más común para aproximar la incertidumbre.

Esta técnica estima un modelo de varianza condicional para las perturbaciones en una variable (inflación o una variable proxy de ella) en lugar de simplemente calcular una medida de variabilidad de resultados pasados (una varianza no condicional). En los modelos GARCH la noción de incertidumbre corresponde a la estimación de una varianza residual que cambia en el tiempo. Aunque en la práctica esta técnica continúa siendo la más utilizada se ha identificado una deficiencia, ya que la varianza condicional de la inflación puede depender tanto de la magnitud como del signo de los choques inflacionarios (Wilson 2006). Por esta razón, en la actualidad, los modelos GARCH asimétricos (GJR-GARCH y E-GARCH) desarrollados por Glosten, Jagannathan y Runkle (1993) y Nelson (1991) se consideran apropiados para estimar la incertidumbre inflacionaria y su asimetría; además de que el efecto de retroalimentación, entre el valor esperado de la inflación y su incertidumbre, se ha estimado a través de la metodología GARCH en media (GARCH-M), desarrollada por Engle, Lilien y Robins (1987).

Como ya se ha mencionado, la adopción del régimen de MI ha buscado atenuar la inflación y su incertidumbre. De acuerdo con Posen (2008), la razón principal, del interés mundial en este esquema, es la falta de un ancla nominal para las expectativas de inflación en la mayoría de los países; razón por la cual, se han movido a un régimen más flexible. El primer país en adoptar el régimen de $\mathrm{Ml}$ fue el Banco de la Reserva de Nueva Zelanda en 1989, seguido por el Banco de Canadá en 1991 y luego por el Banco de Inglaterra en 1992 (Posen, 2008). Muchas economías latinoamericanas y del Sudeste Asiático también han venido adoptando este tipo de políticas; en el primer grupo, los pioneros fueron Chile, Brasil y Colombia en 1999, quienes más tarde fueron imitados por México (2001), Perú (2002) y Uruguay (2007); mientras que, en el segundo grupo la adopción estuvo fuertemente influenciada por las recomendaciones del Fondo Monetario Internacional (FMI), especialmente por aquellos que recibieron paquetes de rescate (cuadro 3 ). 
Cuadro 3. Implementación del régimen de metas de inflación (MI).

\begin{tabular}{|c|c|c|c|c|}
\hline Pais & Fecha de adopción de las MI & $\begin{array}{l}\text { Tasa de inflación a la fecha de } \\
\text { la adopción }\end{array}$ & Tasa de inflación meta & Especificación del objetivo \\
\hline Colombia & Septiembre, 1999 & 9.3 & $2-4 \%$ & $\mathrm{BC}$ \\
\hline México & Enero, 2001 & 9 & $3+/-1 \%$ & $\mathrm{BC}$ \\
\hline Perú & Enero, 2002 & -0.1 & $2+-1 \%$ & $\mathrm{BC}$ \\
\hline Uruguay & Septiembre, 2007 & 9.0 & $3-7 \%$ & $G+B C$ \\
\hline Tailandia & Mayo, 2000 & 0.8 & $0.5-3 \%$ & $\mathrm{BC}$ \\
\hline Corea del Sur & Enero, 2001 & 2.9 & $3+/-1$ & $G+B C$ \\
\hline Filipinas & Enero, 2002 & 4.5 & $4+-1 \%$ & $G+B C$ \\
\hline Indonesia & Julio, 2005 & 7.4 & $5+/-1 \%$ & $G+B C$ \\
\hline
\end{tabular}

Fuente: Elaboración propia con datos de Rogers (2009). Nota: $\mathrm{BC}=$ Banco Central, G= Gobierno.

Para Mishkin (2000:105) las MI son un marco de política monetaria caracterizado por cinco criterios: i) el anuncio público de metas numéricas de mediano plazo para la inflación; ii) un compromiso institucional con la estabilidad de precios como el objetivo principal de la política monetaria; iii) una estrategia de información inclusiva en la que se utilizan muchas variables, no solo los agregados monetarios o el tipo de cambio, para decidir el establecimiento de instrumentos de política; iv) mayor transparencia de la estrategia de política monetaria a través de la comunicación con el público y los mercados sobre los planes, objetivos y decisiones de las autoridades monetarias y v) mayor responsabilidad (rendición de cuentas) del banco central para alcanzar sus MI. Para las economías con mercados emergentes es particularmente importante reconocer estos criterios, ya que, si bien es cierto, los gobiernos de algunos de estos países rutinariamente reportan numéricamente los objetivos de inflación como parte del plan económico de su gobierno; también es cierto que estos no adoptan los demás elementos que son cruciales para el éxito de dicha estrategia.

Se debe considerar que el marco institucional de varias de las economías emergentes, con mucha frecuencia, opera de manera diferente respecto de sus pares en las economías desarrolladas. Para Calvo y Mishkin (2003) son cinco las principales diferencias: i) mayor debilidad en las instituciones fiscales; ii) menor desarrollo de las instituciones financieras (menor regulación-supervisión prudencial gubernamental); iii) baja credibilidad de los bancos centrales; iv) dolarización de pasivos y sustitución de divisas; v) mayor vulnerabilidad a los paros repentinos de los flujos de capital.

Respecto a la primera debilidad, una política fiscal irresponsable presiona a las autoridades monetarias para monetizar la deuda, produciendo así un rápido crecimiento del dinero y una alta inflación. En lo que respecta a la segunda, cuando los mercados identifican la debilidad del sistema bancario se produce una reversión en los flujos de capital (un paro súbito), lo que deriva en una fuerte depreciación del tipo de cambio que, a su vez, conducirá a un incremento en las presiones sobre la tasa de inflación. Además, como resultado de la depreciación, las empresas nacionales verán incrementado el pago de la deuda que se encuentre denominada en moneda 
extranjera (dolarización de pasivos) (Mishkin, 2006). Lo anterior se ha evidenciado en las crisis gemelas (cambiaria y financiera) en Chile 1982, México 1994-95, Este de Asía 1997, Ecuador 1999, Argentina 2001, entre otros.

En lo referente a la tercera debilidad, las economías emergentes carecen de dos acuerdos monetarios fundamentales que son necesarios para permitir a los bancos centrales el control de la inflación: un compromiso público e institucional para la estabilidad de precios, como una meta de largo plazo para la política monetaria y un compromiso para instrumentar la independencia del banco central, esto significa la prohibición del financiamiento directo del déficit gubernamental (Mishkin, 2008). No obstante, en las leyes y constituciones de los países latinoamericanos se establece claramente la conformación de estos acuerdos monetarios. En Colombia, ambos se encuentran consagrados en el artículo 373 de la Constitución Nacional. En México, los dos compromisos se plasman en el artículo 28 de la constitución política de los Estados Unidos Mexicanos. Para el caso de Perú, solamente el primero de estos acuerdos se encuentra en el artículo 2 de la ley orgánica de su banco central. Respecto a Uruguay, el primero de los compromisos se establece en el artículo 3 de la carta orgánica del banco central de Uruguay. En cuanto a las economías del Sudeste Asiático, el banco central de Tailandia establece el primero de los acuerdos en la ley de su banco central de 2008. Mientras que, para Corea del Sur, el primero de los compromisos se establece en la ley del banco de Corea de 2003. En Filipinas, la estabilidad de precios y la prohibición de financiamiento se plasman en las secciones 3 y 128 de la nueva ley de su banco central. Finalmente, en Indonesia, únicamente el primero de los acuerdos se encuentra consagrado en la ley número 23 de 1999 del banco de Indonesia.

La cuarta y quinta debilidad tiene que ver con la mayor preocupación sobre las fluctuaciones de sus tipos de cambio ya que, debido a su historia de baja credibilidad, podría ser más probable que las depreciaciones lleven a un incremento en la tasa de inflación como resultado del traspaso magnificado del tipo de cambio a la inflación.

En particular, el control de la inflación y la estabilización de su incertidumbre es un tema de suma importancia en las regiones de América Latina y el Sudeste Asiático, debido a las altas y volátiles tasas de inflación registradas durante la segunda mitad del siglo pasado. Como resultado de la implementación formal del régimen de $\mathrm{MI}$, entre las principales economías de estas dos regiones, se ha venido desarrollando una creciente cantidad de investigaciones para determinar cuáles han sido los efectos de su aplicación sobre la dinámica de la inflación y su incertidumbre.

Los resultados aún siguen siendo contradictorios. Por un lado, varias investigaciones encuentran que la puesta en marcha de las $\mathrm{Ml}$ es una herramienta útil para la moderación del nivel y la volatilidad de la inflación (Kontonikas, 2004; Vega y Winkelried, 2005; Batini y Laxton 2007; Gonçalves y Salles, 2008; McDermott y McMenamin 2008; Walsh, 2009; Lin y Ye, 2009). En el otro extremo se encuentran las investigaciones que enfatizan las debilidades y limitaciones de esta estrategia para estabilizar la inflación y su incertidumbre y conseguir simultáneamente objetivos macroeconómicos como bajas tasas de desempleo y un alto crecimiento económico (Morón y Winkelried 2005; Capistrán y Ramos-Francia, 2009; Brito y Bystedt, 2010; Carrasco y Ferreiro, 2011).

Después de la crisis financiera global de 2008-2009 se han agudizado las continuas críticas. La idea de que el régimen de MI aumentaría la credibilidad del banco central y que con ello se po- 
dría alcanzar un mejor desempeño en los fundamentos macroeconómicos (un mayor crecimiento del producto, menores tasas de desempleo, mayor inversión de capital) sigue siendo controvertida tanto en la teoría como en la práctica.

\section{Discusión sobre la aplicación del régimen de MI}

Si bien es cierto que el valor esperado de la tasa de inflación junto con su incertidumbre se ha atenuado en las recientes décadas, y esto se ha visto como un posible éxito atribuible al régimen de $\mathrm{Ml}$, también es cierto que, en ambas regiones, se identifican características de suma relevancia al momento de poner en práctica las políticas monetarias. Comenzando por el hecho de que, en el primer grupo de países, la especificación del objetivo de meta de inflación queda a cargo, exclusivamente, del banco central en tres de las cuatro economías; mientras que, en el caso de las economías del Sudeste Asiático, la responsabilidad la comparten el Gobierno y el instituto central en tres de las cuatro economías analizadas.

Los resultados de los principales objetivos macroeconómicos, como el crecimiento económico, la inversión en capital o la disminución del desempleo, también han sido muy diferentes. Las economías del Sudeste Asiático han tenido un mejor desempeño, tal como lo muestra el cuadro 4. En ella se identifican los principales agregados macroeconómicos. En la segunda columna del cuadro se puede apreciar que la región del Sudeste Asiático ha mantenido tasas de crecimiento del producto superiores a las observadas en América Latina. En la tercera columna los resultados sobre la disminución de las tasas de desempleo también le son favorables a la región asiática, pues han logrado mantener bajas tasas de desocupación antes y después de la implementación del régimen de MI. Otro rubro que evidencia el boyante desempeño de esta región se presenta en el comercio, que se ha visto duplicado en Tailandia y Corea después de la adopción de este esquema monetario. En América Latina tal situación solamente se observa en el caso de México.

Finalmente, en la columna que presenta las tasas de crecimiento de la inversión como porcentaje del Producto Interno Bruto, una vez más la región del Sudeste Asiático ha tenido un mayor crecimiento en este rubro, antes y después de la implementación del paradigma de MI.

Cuadro 4. Agregados macroeconómicos para Latinoamérica y el Sudeste Asiático

\begin{tabular}{|c|c|c|c|c|c|c|c|c|c|}
\hline \multirow[b]{2}{*}{ País } & \multirow[b]{2}{*}{$\begin{array}{c}\text { Año de imple- } \\
\text { mentación de } \\
\text { la MI }\end{array}$} & \multicolumn{2}{|c|}{$\begin{array}{c}\text { Tasa de crecimiento del } \\
\text { Producto }^{1}\end{array}$} & \multicolumn{2}{|c|}{ Tasa de desempleo ${ }^{2}$} & \multicolumn{2}{|c|}{$\begin{array}{l}\text { Comercio: Exportaciones + } \\
\text { Importaciones }(\% \text { PIB })^{3}\end{array}$} & \multicolumn{2}{|c|}{$\begin{array}{l}\text { Formación bruta de capital } \\
\qquad(\% \text { PIB })^{4}\end{array}$} \\
\hline & & Antes $\mathrm{MI}$ & Después MI & Después MI & Antes $\mathrm{MI}$ & Antes $\mathrm{MI}$ & Después MI & Antes MI & Después MI \\
\hline Colombia & 1999 & 4.26 & 4.00 & 11.48 & 11.79 & 29.81 & 36.11 & 19.31 & 21.84 \\
\hline México & 2001 & 4.80 & 2.05 & 3.84 & 4.05 & 27.46 & 60.89 & 21.69 & 22.57 \\
\hline Perú & 2002 & 3.01 & 5.36 & 5.70 & 3.94 & 34.95 & 47.40 & 26.62 & 21.71 \\
\hline Uruguay & 2007 & 1.98 & 4.05 & 11.53 & 7.11 & 37.20 & 52.32 & 16.77 & 20.29 \\
\hline Tailandia & 2000 & 6.93 & 4.03 & 1.82 & 1.21 & 54.44 & 128.25 & 27.77 & 25.19 \\
\hline Corea & 2001 & 8.94 & 3.84 & 3.47 & 3.51 & 46.97 & 84.02 & 29.65 & 31.12 \\
\hline
\end{tabular}




\begin{tabular}{|l|l|l|l|l|l|l|l|l|l|}
\hline Filipinas & 2002 & 3.86 & 5.50 & 3.77 & 3.46 & 55.06 & 78.26 & 23.23 & 20.75 \\
\hline Indonesia & 2005 & 5.18 & 5.53 & 4.98 & 5.44 & 45.52 & 48.13 & 22.80 & 31.65 \\
\hline
\end{tabular}

Fuente: Elaboración propia con información de las Estadísticas Financieras Internacionales (FMI) y datos del Banco Mundial. Notas: ${ }^{1}$ periodo 1960-2017, ${ }^{2}$ periodo 1991-2017, ${ }^{3}$ periodo 1960-2017, ${ }^{4}$ periodo 1960-2017. En todos los casos se dividió en dos muestras, separadas cada una por la fecha de implementación del régimen de MI.

En el cuadro 5 se presentan los principales precios de las economías seleccionadas. Se aprecia cómo, antes de la implementación del régimen de MI, las economías sufrieron periodos de alta inflación, inclusive de hiperinflación: Perú (7481.6\% en 1990). Lo anterior llevó a la instrumentación de un proceso de ajuste, siguiendo las recomendaciones del consenso de Washington, que condujo a una tendencia decreciente de los precios, alcanzando tasas de inflación de un digito en el periodo posterior al régimen de $\mathrm{MI}$.

Cuadro 5. Inflación, tasa de interés y reservas internacionales para Latinoamérica y el Sudeste Asiático

\begin{tabular}{|c|c|c|c|c|c|c|c|}
\hline \multicolumn{2}{|c|}{} & \multicolumn{2}{|c|}{ Tasa de inflación $^{1}$} & \multicolumn{2}{c|}{${\text { Tasa de interés del Banco Central }{ }^{2}}^{2}$} & \multicolumn{2}{c|}{ Reservas internacionales del BC (millo- $^{\text {nes USD) }^{3}}{ }^{3}$} \\
\hline País & $\begin{array}{c}\text { Año de implemen- } \\
\text { tación de la MI }\end{array}$ & Antes MI & Después MI & Antes MI & Después MI & Antes MI & Después MI \\
\hline Colombia & 1999 & 19.05 & 4.94 & 40.31 & 14.10 & 3397.44 & 26723.23 \\
\hline México & 2001 & 26.29 & 4.33 & 29.18 & 6.71 & 8034.53 & 117372.34 \\
\hline Perú & 2002 & 315.72 & 2.79 & 31.32 & 21.70 & 2746.00 & 39330.48 \\
\hline Uruguay & 2007 & 46.44 & 7.98 & 50.65 & 13.28 & 1325.47 & 12486.00 \\
\hline Tailandia & 2000 & 5.25 & 2.14 & 13.36 & 5.02 & 8636.83 & 113120.31 \\
\hline Corea & 2001 & 10.42 & 2.60 & 11.27 & 5.50 & 11489.73 & 266132.50 \\
\hline Filipinas & 2002 & 10.64 & 3.73 & 16.67 & 7.67 & 3480.15 & 52080.64 \\
\hline Indonesia & 2005 & 56.39 & 6.28 & 20.78 & 12.93 & 12285.40 & 91683.39 \\
\hline
\end{tabular}

Fuente: Elaboración propia con información de las Estadísticas Financieras Internacionales (FMI) y datos del Banco Mundial. Notas: ${ }^{1}$ periodo $1960-2017,{ }^{2}$ periodo $1986-2017,{ }^{3}$ periodo $1960-2017$.

Entre los requisitos establecidos por el régimen de $\mathrm{Ml}$, se encuentra la implementación de un sistema de tipo de cambio flexible en el contexto de libre movilidad de capitales. En este escenario, la política de los bancos centrales se ha orientado a disminuir la tasa de interés sustancialmente como un instrumento anticíclico. Lo que resulta desconcertante es la rápida y significativa expansión de las reservas internacionales que exhiben todas las economías de ambas muestras.

Esta acumulación fue especialmente pronunciada en México, Tailandia, Corea e Indonesia. El fenómeno resulta enigmático, ya que la celebrada flexibilidad del régimen cambiario se defendió, precisamente, con el argumento de que bajo el esquema de $\mathrm{MI}$, los bancos centrales obtendrían libertad en sus políticas monetarias y no necesitarían mantener reservas en moneda extranjera para defender la tasa objetivo de sus tipos de cambio. 
Los proponentes del régimen de $\mathrm{Ml}$ argumentan que la necesidad de acumular estas reservas deriva del mantenimiento de la estabilidad de precios en contra de posibles choques. Sin embargo, la emblemática defensa de la estabilidad de precios se ha logrado, en gran parte, a expensas de grandes y costosas reservas internacionales que se mantienen prácticamente inactivas en las arcas de los bancos centrales, lo que resulta muy cuestionable en una época de desempleo prolongado y lento crecimiento de la inversión (Epstein y Yeldan, 2008:138).

Lo que se ha visto en tiempos de crisis es que los bancos centrales de las economías emergentes, principalmente las del Sudeste Asiático, han utilizado herramientas de política no convencionales, las cuales se desvían del paradigma de MI (Epstein y Yeldan, 2008; Lim, 2008), entre las principales podemos mencionar: 1) El mantenimiento de un tipo de cambio real competitivo, como en Corea del Sur; 2) La implementación de técnicas de controles de capital (impuestos sobre los flujos de capital de corto plazo), como en Malasia y Chile, que mantengan un equilibrio entre las tasas de internas y externas y que tienen la capacidad de evitar crisis monetarias (modificaciones a gran escala en los tipos de cambio); además de proporcionar autonomía a la expansión de los bancos centrales y prevenir la apreciación de las monedas; 3) Una declaración explícita para las metas de crecimiento del producto, el empleo y la inversión; 4) Políticas antimonopolio para limitar la inflación a niveles moderados y 5) Programas de crédito focalizado, especialmente para las pequeñas y medianas empresas orientadas a la exportación, y que pueden contribuir al crecimiento de la productividad y el empleo.

\section{Metodología econométrica para identificar la naturaleza de la inflación y su incertidumbre}

La tasa de inflación se puede aproximar mediante la diferencia logarítmica del índice de precios al consumidor, también definida como la tasa de cambio porcentual del índice de precios al consumidor, es decir, $\pi_{t}=\left(\log \left(I P C_{t}\right)-\log \left(I P C_{t-1}\right)\right) * 100 \approx\left(\frac{I P C_{t}-I P C_{t-1}}{I P C_{t-1}}\right) * 100$.

En el caso de la infiacıon, Igua que eı ae ı mayorıa ae ıas serıes de tiempo económicas no poseen una varianza constante, y algunas de ellas exhiben fases de relativa tranquilidad seguida por periodos de alta volatilidad (Enders, 2015:118). En los modelos econométricos convencionales, la varianza del término de perturbación se asume como una constante; sin embargo, cuando dicha varianza es heterocedástica, la incertidumbre tiende a agruparse en periodos de alta y baja volatilidad.

Comúnmente el término de volatilidad se ha asociado con la varianza del término de perturbación; aunque no existe una definición concreta y generalizada de volatilidad, se acepta ampliamente que esta se refiere a un periodo en el cual la serie de tiempo presenta alta variabilidad o una varianza creciente (Baillie, Chung y Tieslau, 1996). De acuerdo con Castillo y Ramoni-Perazzi (2017), podemos identificar que la volatilidad, también entendida como incertidumbre, se caracteriza por: i) Exceso de curtosis (Mandelbrot, 1963; Fama, 1963); ii) persistencia o conglomerado de volatilidad (Mandelbrot, 1963, Engle, 1982); iii) discontinuidades en la serie (Figlewski, 1997); iv) comportamientos asimétricos de las series temporales (Campbell, 1987; Hentschel,1995) y v) 
Efecto apalancamiento. ${ }^{1}$ La estructura de ecuaciones que capture la media y varianza condicional de la tasa de cambio porcentual del índice de precios al consumidor (inflación), además de abordar las características antes mencionadas debe plantear una estructura que sea capaz de estimar adecuadamente las funciones no lineales.

\subsection{Modelos SARIMA}

Para poder identificar el valor esperado o media condicional de la tasa de inflación se debe especificar una ecuación adecuada, para lo cual se propone una ecuación en diferencias estocástica, $\operatorname{ARMA}(p, q)$ representada como:

$$
\pi_{t}=a_{0}+\sum_{i=1}^{p} a_{i} \pi_{t-i}+\sum_{i=0}^{q} B_{i} \varepsilon_{t-i}
$$

La ecuación [1] representa un modelo autorregresivo de medias móviles (ARMA), cuyas raíces características son menores a la unidad, con lo que se garantiza que el proceso sea estacionario. Sin embargo, la inflación ha demostrado poseer una clara variación estacional, por lo que su comportamiento debe ser incluido en la ecuación estocástica, es decir, debe ser representada con un modelo multiplicativo que contenga el componente estacional SARIMA $(p, d, q) x(P, D, Q)_{m^{\prime}}$ con " $m$ " representando la estacionalidad (Hyndman y Athanasopoulos, 2018).

$$
=\gamma_{0}+\sum_{i=1}^{p} \gamma_{i} \pi_{t-i}+\sum_{i=0}^{q} \theta_{i} \varepsilon_{t-i}+\sum_{i=1}^{P} \phi_{i} \pi_{t-i s}+\sum_{i=1}^{Q} \delta_{i} \varepsilon_{t-i s}+\varepsilon_{t}
$$

En la ecuación anterior, $\pi$ es la tasa de cambio porcentual del índice de precios al consumidor (inflación) ; mientras que, $\pi_{(t-i)}$ son sus valores rezagados, $\varepsilon_{t-i}$ representa las medias moviles ordinarias, $\pi_{(t-i s)}$ son los valores autorregresivos estacionales de longitud $s, \varepsilon_{t-i s}$ representa las medias moviles estacionales $y$, finalmente, $\varepsilon_{t}$ es el término de perturbación estocástica que se asume como ruido blanco. Estos modelos presentan una gran gama de posibilidades para identificar la correcta especificación de la media condicional de la inflación (Grier y Perry 2000; Grier y Grier, 2006; Daal, Naka y Sánchez, 2005; Caballero y Caballero 2015). De esta manera, se puede interpretar un modelo $\mathrm{AR}(1)$ y $\mathrm{MA}(1)$, ambos con componentes ordinario (no estacional) y estacional mensual.

Figura 1. Modelo autorregresivo integrado y de media móvil estacional

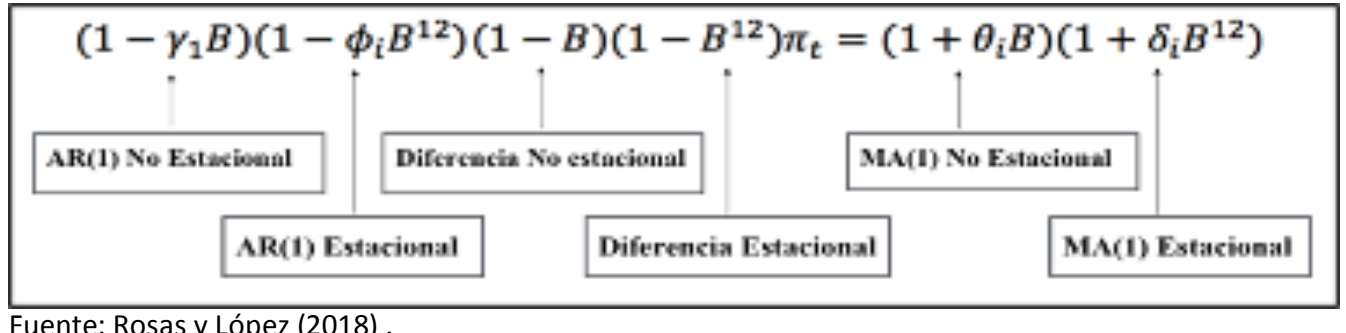

Fuente: Rosas y López (2018) .

\footnotetext{
${ }^{1}$ Este efecto se refiere al hecho de que los cambios en los precios de las variables tienden a correlacionarse negativamente con los cambios en la volatilidad (López, 2004), por ejemplo, esto indicaría que un aumento en el riesgo de un activo financiero conduciría a una disminución de su valor.
} 
A través de la metodología Box-Jenkins se obtiene el proceso SARIMA (autorregresivos integrados de medias móviles estacionales), que identifique el valor esperado de la inflación. Se trata de un proceso iterativo de etapas correspondientes a la selección de modelos, estimación de parámetros y verificación de los mismos. Las explicaciones recientes del proceso a menudo añaden una etapa preliminar de preparación de datos y una etapa final de aplicación del modelo (pronóstico) (Makridakis, Wheelwright y Hyndman, 1998).

\subsection{Modelos ARCH y GARCH}

Al tomar como referencia la estructura de Rosas y Mimbrera (2018), una vez que se ha especificado la media condicional de la tasa de cambio porcentual del índice de precios al consumidor, el siguiente objetivo es modelar la varianza condicional e identificar las relaciones de causalidad simultánea entre la media condicional de la inflación y su varianza condicional (la incertidumbre inflacionaria). Dado que $E_{t}\left(\varepsilon_{t+1}\right)^{2}$, se puede modelar como un proceso $A R(q)$, utilizando las perturbaciones cuadráticas estimadas, es decir:

$$
=\alpha_{0}+\alpha_{1} \hat{\varepsilon}_{t-1}^{2}+\alpha_{2} \hat{\varepsilon}_{t-2}^{2}+\cdots+\alpha_{q} \hat{\varepsilon}_{t-q}^{2}+V_{t}
$$

Por esta razón, una ecuación como la [3] se denomina modelo autorregresivo de orden " $q$ " en los residuales cuadrados.

Engle (1982) mostró que es posible modelar, simultáneamente, la media y la varianza de una serie de tiempo. Dado un modelo de regresión lineal estándar:

$$
\begin{gathered}
Y_{t}=\beta^{\prime} X_{t}+\varepsilon_{t} \\
\varepsilon_{t}=v_{t} \sqrt{\alpha_{0}+\alpha_{1} \varepsilon_{t-1}^{2}}
\end{gathered}
$$

En donde $v_{t}$ es un proceso de ruido blanco en el que $\sigma_{v}^{2}=1$, y $v_{t}$ y $\varepsilon_{t-1}$ son independientes el uno del otro.

Además, los parámetros de $\alpha_{0}$ y $\alpha_{1}$ y deben ser constantes tales que $\alpha_{0}>0$ y $0 \leq \alpha_{1} \leq 1$

siendo esta última condición la que asegura que el proceso sea estable. En 1986 Bollerslev extendió el trabajo original de Engle al desarrollar una técnica que permite que la varianza condicional sea un proceso ARMA.

En esta propuesta el error de dicho proceso se encuentra definido como:

$\varepsilon_{t}=v_{t} \sqrt{h_{t}}$; donde $\sigma_{v}^{2}=1 h_{t}=E_{t-1}\left(\varepsilon_{t}\right)^{2}$. Al ser un proceso ruido blanco, su media condicional es igual a cero. Por su parte, la varianza condicional es $E_{t-1} \varepsilon_{t}^{2}=h_{t} \quad$ (incertidumbre inflacionaria), donde la varianza condicional de es un proceso ARMA dado por . Así, el modelo GARCH se define como:

$$
h_{t}=\alpha_{0}+\sum_{i=1}^{q} \alpha_{i} \varepsilon_{t-i}^{2}+\sum_{i=1}^{p} \beta_{i} h_{t-i}
$$




$$
h_{t}=\alpha_{0}+A(L) \varepsilon_{t}^{2}+B(L) h_{t}
$$

Con $p \geq 0, q>0, \alpha_{0}>0, \alpha_{i} \geq 0, \beta_{i} \geq 0$. En el proceso $\mathrm{ARCH}(\mathrm{q})$, la varianza condicional únicamente es especificada como una función lineal de las varianzas muestrales pasadas; mientras que, en el proceso GARCH ( $p, q)$, se permite la inclusión de varianzas condicionales rezagadas. De acuerdo con Bollerslev (1986), el proceso GARCH (p, q) como se define en [9] es estacionario en sentido amplio, con $E_{t}\left(\varepsilon_{t}\right)=0, \operatorname{Var}\left(\varepsilon_{t}\right)=\alpha_{0}(1-A(1)-B(1))^{(-1)}$ y $\operatorname{Cov}\left(\varepsilon_{t}, \varepsilon_{s}\right)=0$ para $\mathrm{t} \neq \mathrm{s}$ si y sólo si $A(1)+B(1)<1$. Esta condición de estacionariedad es importante para asegurar que los momentos de la distribución normal sean finitos.

El beneficio de un modelo GARCH consiste en que los modelos $A R C H$ de orden superior pueden tener una representación GARCH más parsimoniosa, por ejemplo, un modelo GARCH $(1,1)$ puede tener una representación como la de un $\mathrm{ARCH}(\infty)^{2}$, esto lo vuelve más fácil de identificar y de estimar, además de que implica menos restricciones a los coeficientes (Posedel, 2005). Igual que un proceso SARIMA es identificado con sus funciones de Autocorrelación simple (FAC) y parcial (FACP) del término de perturbación, la FACP de los residuales cuadráticos puede ayudar a identificar el orden del proceso GARCH, para ello se utiliza una prueba de multiplicador lagrangiano (McLeod y Li, 1983).

En la literatura sobre inflación e incertidumbre inflacionaria se han adoptado modelos econométricos que permiten estimar, simultáneamente, el valor esperado de la inflación y su incertidumbre inflacionaria (Grier y Perry, 2000; Fountas, 2010; Perrotini y Rodríguez, 2012). Estos modelos, denominados GARCH en media (GARCH-M) permiten la comprobación simultanea de las hipótesis establecidas. A continuación, se presentan los modelos de media y varianza condicional de la inflación mediante la estructura $\operatorname{SARIMA}(p, d, q)(P, D, Q)_{s}-G A R C H(p, q)-M$. Para la representación de la media y la varianza condicional el sistema de ecuaciones queda establecido como:

$$
\pi_{t}=\gamma_{0}+\sum_{i=1}^{p} \gamma_{i} \pi_{t-i}+\sum_{i=0}^{q} \theta_{i} \varepsilon_{t-i}+\sum_{i=1}^{P} \phi_{i} \pi_{t-i s}+\sum_{i=1}^{Q} \delta_{i} \varepsilon_{t-i s}+\varphi \sqrt{h_{t}}+\varepsilon_{t}
$$

${ }^{2} U n$ proceso $\operatorname{GARCH}(1,1)$ se puede representar como un proceso $\mathrm{ARCH}(\infty)$.

$$
\begin{gathered}
\sigma^{2}{ }_{t}=\alpha_{0}+\alpha_{1} \varepsilon_{t-1}^{2}+\beta_{1} \sigma^{2}{ }_{t-1} \\
\sigma^{2}{ }_{t}=\alpha_{0}+\alpha_{1} \varepsilon_{t-1}^{2}+\beta_{1}\left(\alpha_{0}+\alpha_{1} \varepsilon_{t-2}^{2}+\beta_{1} \sigma^{2}{ }_{t-2}\right) \\
\sigma^{2}{ }_{t}=\alpha_{0}+\alpha_{1} \varepsilon_{t-1}^{2}+\alpha_{0} \beta_{1}+\alpha_{1} \beta_{1} \varepsilon_{t-2}^{2}+\beta_{1}{ }^{2} \sigma^{2}{ }_{t-2} \\
\sigma^{2}{ }_{t}=\alpha_{0}+\alpha_{1} \varepsilon_{t-1}^{2}+\alpha_{0} \beta_{1}+\alpha_{1} \beta_{1} \varepsilon_{t-2}^{2}+\beta_{1}{ }^{2}\left(\alpha_{0}+\alpha_{1} \varepsilon_{t-3}^{2}+\beta_{1} \sigma^{2}{ }_{t-3}\right) \\
\vdots \\
\sigma^{2}{ }_{t}=\frac{\alpha_{0}}{1-\beta_{1}}+\alpha_{1} \sum_{i=0}^{\infty} \varepsilon_{t-1-i}{ }^{2} \beta_{1}{ }^{i}
\end{gathered}
$$




$$
h_{t}=\alpha_{0}+\sum_{i=1}^{q} \alpha_{i} \varepsilon_{t-i}^{2}+\sum_{i=1}^{p} \beta_{i} h_{t-i}+\delta \pi_{t-i}+\theta_{1} D M I_{t}+\theta_{2} D M I_{t} * \pi_{t-1}
$$

Donde $\alpha_{0}$ es la varianza condicional media, $\varepsilon^{2}{ }_{(t-i)}$ los choques sobre la incertidumbre inflacionaria pasados, $\mathrm{h}_{(t-i)}$ las varianzas condicionales pasadas y $\varepsilon_{\mathrm{t}}$ el término de perturbación aleatoria, ruido blanco. De manera simultánea se aplica la estructura GARCH en media (GARCH-M) que permite incluir en la media su propia desviación estándar condicional $\left(\sqrt{h_{t}}=\frac{\varepsilon_{t}}{v_{t}}\right)$ cuya influencia (efecto marginal) está captado por el coeficiente $\varphi$ y de la inflación rezagada un periodo, cuyo efecto marginal está captado por el coeficiente $\delta$. Adicionalmente, se agregaron variables binarias para capturar el efecto de la implementación del régimen de $\mathrm{Ml}$ en la incertidumbre inflacionaria, así como del efecto de los cambios estructurales. ${ }^{3}$

Al considerar que los modelos de heterocedasticidad condicional mantienen una restricción de simetría sobre la incertidumbre, se propone la ampliación de la ecuación de varianza condicional a un esquema GJR-GARCH (Zakoïan 1994; Glosten, Jagannathan y Runkle, 1993); cuya estructura identifica la magnitud de la influencia de los choques positivos y negativos. De esta manera, la ecuación [9] se transforma en [9a].

$$
\begin{gathered}
h_{t}=w+\alpha \varepsilon_{t-1}^{2}+\gamma \varepsilon^{2}{ }_{t-1} * I_{t-1}+\beta h_{t-1}+\delta \pi_{t-i}+\theta_{1} D M I_{t}+\theta_{2} D M I_{t} * \pi_{t-1} \\
I_{t-1}=\left\{\begin{array}{c}
1 \text { si } \varepsilon_{t-1}<0 \\
0 \text { en otro caso }
\end{array}\right.
\end{gathered}
$$

Con $\omega$ representando el intercepto, $\alpha, \gamma$ y $\beta$ son parámetros positivos y el proceso es convergente siempre y cuando se garantice la condición: $\alpha+\beta<1$. En este esquema los choques positivos $\varepsilon_{(t-1)}>0$ (malas noticias) y los choques negativos $\varepsilon_{(t-1)}<0$ (buenas noticias) tienen diferentes efectos sobre la incertidumbre inflacionaria. De esta manera, si $\gamma_{i} \neq 0$, el impacto de las noticias será asimétrico. De igual forma, su estructura incluye las variables binarias para capturar los efectos ya señalados.

\section{Estimación y resultados empíricos}

En la implementación de la metodología Box-Jenkins se debe analizar la serie de tiempo para determinar la presencia de una posible raíz unitaria en la inflación (). Para determinar si estadísticamente la inflación es una serie de tiempo estacionaria o no, se implementan las pruebas de raíz unitaria Dickey-Fuller Aumentada (ADF, por sus siglas en inglés) y Phillips-Perron (PP). En el cuadro

${ }^{3}$ De acuerdo con Enders (2015: 227), cuando existen cambios estructurales en las series de tiempo las pruebas estadísticas DickeyFuller para estacionariedad se encuentran sesgadas hacia no rechazar una raíz unitaria, es decir, no se rechaza que la serie sea no estacionaria. Para subsanar esta deficiencia Vogelsang y Perron (1998) propusieron una clase de estadísticos de prueba que permiten dos formas diferentes de quiebre estructural. Estos son los modelos Additive Outlier (AO) e Innovational Outlier (IO). 
6 se presentan los estadísticos de las pruebas de raíz unitaria para la inflación $(\pi)$, se comprueba que la serie transformada con intercepto y tendencia e intercepto son estadísticamente significativos, lo que demuestra que la serie de tiempo es estacionaria (columnas 2 a 5).

Adicionalmente, se aplicó la prueba de Vogelsang y Perron (1998), la cuál es capaz de identificar la existencia de una raíz unitaria en presencia de un cambio estructural en la constante y en la tendencia e intercepto (columnas 6 a 7 del cuadro 6). En estas columnas se puede identificar que todas las series de tiempo presentan la propiedad de estacionariedad.

Cuadro 6. Pruebas de raíz unitaria y cambio estructural sobre la inflación

\begin{tabular}{|c|c|c|c|c|c|c|}
\hline \multirow{3}{*}{ PAIS } & & & & & \multicolumn{2}{|c|}{ Vogelsang y Perron } \\
\hline & \multicolumn{2}{|c|}{$A D F$} & \multicolumn{2}{|c|}{$P P$} & \multicolumn{2}{|c|}{ Modelo quiebres en $C y T$} \\
\hline & c & CyT & $c$ & CyT & $t$-stat & Fecha quiebre \\
\hline COLOMBIA & -2.428 & -2.973 & $-10.407 * * *$ & $-11.328^{* * *}$ & $-6.195 * * *$ & 1998.04 \\
\hline MEXICO & $-4.152^{* * *}$ & $-4.181^{* * *}$ & $-6.110^{* * *}$ & $-6.149 * * *$ & $-7.881^{* * *}$ & 1988.01 \\
\hline PERU & $-4.645^{* * *}$ & $-4.662^{* * *}$ & $-17.927^{* * *}$ & $-17.945^{* * *}$ & $-14.387^{* * *}$ & 1990.08 \\
\hline URUGUAY & $-3.590 * * *$ & $-22.887^{* * *}$ & $-17.927 * * *$ & $-24.047 * * *$ & $-4.828^{* *}$ & 1995.01 \\
\hline TAILANDIA & $-9.469 * * *$ & $-9.893 * * *$ & $-19.883 * * *$ & $-19.764^{* * *}$ & $-6.320 * * *$ & 1981M03 \\
\hline COREA & -2.761 & $-13.757 * * *$ & $-12.573 * * *$ & $-13.662 * * *$ & $-6.529 * * *$ & 1981M06 \\
\hline FILIPINAS & $-6.023^{* * *}$ & $-6.167 * * *$ & $-18.592 * * *$ & $-18.595 * * *$ & $-8.509 * * *$ & 1984M07 \\
\hline INDONESIA & $-10.486^{* * *}$ & $-10.832 * * *$ & $-23.570 * * *$ & $-23.610^{* * *}$ & $-6.738 * * *$ & 1997M06 \\
\hline
\end{tabular}

Fuente: Elaboración propia. Nota: Para determinar el número de términos en diferencia rezagados se utilizó el criterio de información de Schwarz para la prueba DFA y el criterio de Newey-West Bandwith para la prueba PP. Los asteriscos ***, **, * indican el rechazo de la hipótesis nula (no estacionariedad) a un nivel de significancia de 1, 5 y 10 por ciento, respectivamente.

En los cuadros (7) y (8) se muestran los resultados de la estimación de las ecuaciones [8] y [9a], la ecuación de la media condicional y la varianza condicional, respectivamente; se trata de una especificación. Para la estimación de los coeficientes de cada uno de los modelos correspondientes a los países latinoamericanos y del Sudeste Asiático se utilizó la metodología de cuasimáxima verosimilitud $\mathrm{ARCH}$ y la técnica de optimización numérica de Berndt et al. (1974), también conocido como el algoritmo $\mathrm{BHHH}$.

Como se observa, la ecuación de la media condicional () muestra que los valores autorregresivos y de medias móviles, ordinarios y estacionales, se ajustan estadísticamente en cada una de las ecuaciones. Respecto al coeficiente, que mide el efecto marginal de la desviación estándar condicional (volatilidad) sobre la inflación, los valores estimados son positivos y los coeficientes estadísticamente significativos para México, Perú, Uruguay, Corea del Sur, Filipinas, Indonesia y Tailandia; lo que revela el cumplimiento de la hipótesis de Cukierman y Meltzer, es decir, los niveles de incertidumbre inflacionaria a la baja conducen a tasas de inflación más bajas. 
Cuadro 7. Resultados de las regresiones GJR-GARCH(1,1)-M para América Latina

\begin{tabular}{|c|c|c|c|}
\hline País & $\begin{array}{l}\pi_{t}=\gamma_{0}+\sum_{i=1}^{D} \gamma_{i} \pi_{t-i}+\sum_{i=1}^{Q} \theta_{i} \varepsilon_{t-i}+\sum_{i=1}^{P} \phi_{i} \pi_{t-i s}+\sum_{t=1}^{Q} \delta_{i} \varepsilon_{t-i s}+\varphi \sqrt{h_{t}}+\varepsilon_{t} \\
h_{t}=w+\alpha \varepsilon_{t-1}^{2}+\gamma \varepsilon_{t-1}^{2} \cdot I_{t-1}+\beta h_{t-1}+\delta \pi_{t-1}+\theta_{1} D M I_{t}+\theta_{2} D M I_{t} \cdot \pi_{t-1}\end{array}$ & $R^{2}$ & $L L$ \\
\hline \multirow{2}{*}{ Colombia } & 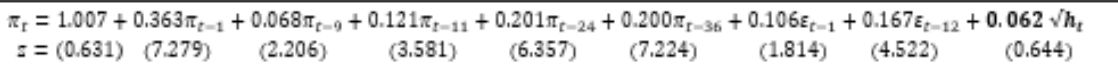 & 0.636 & -437.7 \\
\hline & 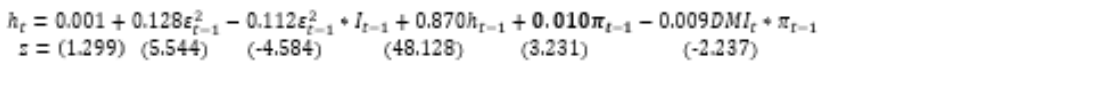 & & \\
\hline \multirow{2}{*}{ México } & 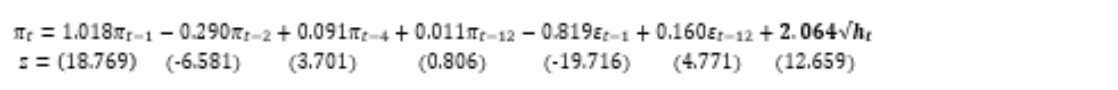 & 0.722 & 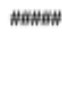 \\
\hline & 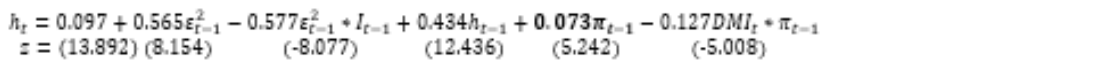 & & \\
\hline \multirow{2}{*}{ Perú } & 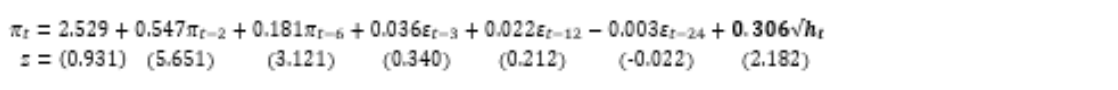 & 0.288 & -1795 \\
\hline & 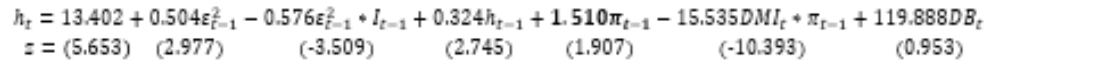 & & \\
\hline \multirow{2}{*}{ Uruguay } & 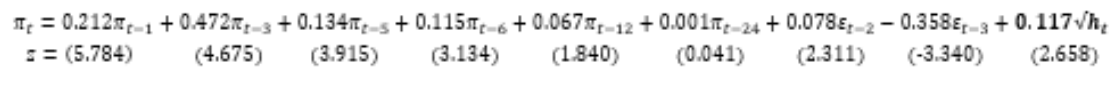 & 0.433 & -1274 \\
\hline & 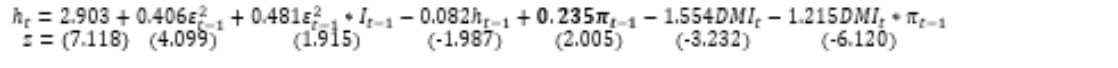 & & \\
\hline
\end{tabular}

Fuente: Elaboración propia

En lo que respecta a la ecuación de la varianza condicional $\left(h_{t}\right)$, el efecto de la inflación rezagada, $\left(\pi_{t-1}\right)$ sobre la varianza en $t$, denotada por el coeficiente $\delta$, presenta evidencia mixta respecto a las hipótesis planteadas. Se identifica un valor positivo y estadísticamente significativo para el coeficiente de la inflación rezagada un periodo para el caso de Colombia, México, Perú, Uruguay, Filipinas y Tailandia, lo que evidencia el cumplimiento de la hipótesis de Friedman-Ball; mientras que, para el caso de Corea del Sur, la evidencia es favorable a la hipótesis de Pourgerami - Maskus a un nivel de significancia de $10 \%$ y para Indonesia no hay significancia estadística. Respecto a la ecuación [9a], los resultados señalan que la condición de estacionariedad $\alpha_{1}+\beta_{1}<1$ se verifica en todos los modelos de la varianza condicional $\left(h_{t}\right)$, con lo que se garantiza que su trayectoría tiende a converger a su valor medio en el largo plazo. 
Cuadro 8. Resultados de las regresiones GJR-GARCH(1,1)-M para el Sudeste Asiático

\begin{tabular}{|c|c|c|c|}
\hline & 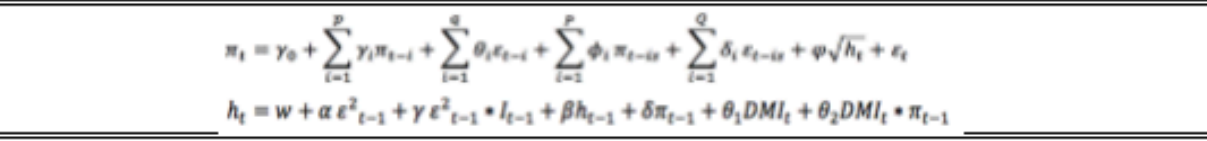 & $R^{2}$ & $L L$ \\
\hline \multirow{2}{*}{ Corea } & 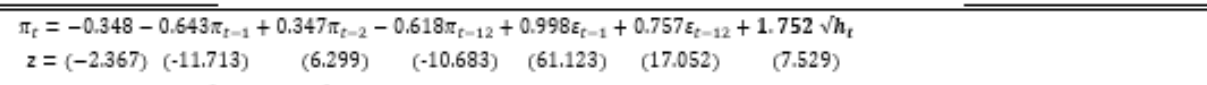 & 0.430 & -43557 \\
\hline & $\begin{aligned} h_{t} & =0.057+0.173 \varepsilon_{t-1}^{2}+0.069 \varepsilon_{t-1}^{2} * I_{t-1}+0.6067 h_{t-1}-0.059 \pi_{t-1}-0.270 D B_{t}-0.127 D M I_{t} * \pi_{t-1} \\
\varepsilon & =(5.227)(3.463) \quad(0.776) \quad(8.755) \quad(-1.778) \quad(-3.521)\end{aligned}$ & & \\
\hline \multirow[b]{2}{*}{ Filipinas } & $\begin{array}{l}\pi_{t}=0.361+0.301 \pi_{\mathrm{r}-1}+0.015 \pi_{t-4}+0.173 \pi_{t-12}+0.316 \sqrt{ } h_{t} \\
z=(6.029)(7.022) \quad(0.4086) \quad(8.013) \quad(3.141)\end{array}$ & 0.275 & -777.7 \\
\hline & 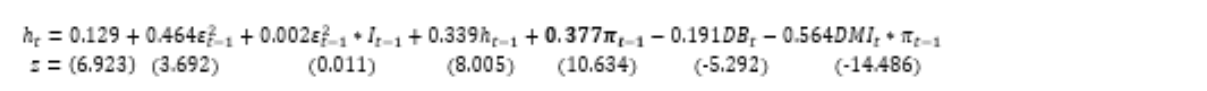 & & \\
\hline \multirow{2}{*}{ Indonesia } & $\begin{aligned} \pi_{t} & =0.024+0.145 \pi_{t-1}+0.018 \pi_{\mathrm{r}-4}+0.050 \pi_{t-6}+0.135 \pi_{t-12}-0.003 \varepsilon_{t-12}+1.017 \sqrt{ } h_{t} \\
z & =\left(\begin{array}{llllll}0.329) & (3.572) & (0.696) & (2.385) & (3.517) & (-0.078)\end{array}\right.\end{aligned}$ & 0.197 & 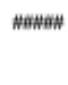 \\
\hline & 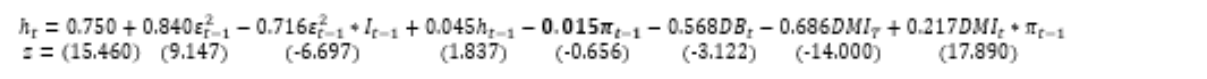 & & \\
\hline \multirow{2}{*}{ Tailandia } & $\begin{aligned} & \pi_{t}=0.180+0.207 \pi_{t-1}+0.101 \pi_{t-3}+0.070 \pi_{t-3}+0.066 \pi_{r-12}+0.137 \pi_{t-36}-0.027 \varepsilon_{t-2}+0.121 \varepsilon_{r-12}+0.058 \varepsilon_{t-24}+0.288 \sqrt{ } h_{t} \\
& z=(2.689) \quad(3.476) \quad(2.334) \quad(1.646) \\
&\end{aligned}$ & 0.259 & -434.9 \\
\hline & 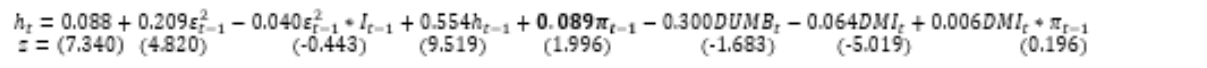 & & \\
\hline
\end{tabular}

Fuente: Elaboración propia

Siguiendo con la ecuación [9a], se observa que el estimador correspondiente al componente del umbral $\left(\varepsilon_{t-1}^{2}{ }^{*} I_{t-1}\right)$ es negativo y estadísticamente significativo para Colombia, México, Perú e Indonesia (efecto apalancamiento inverso), en tanto que para Uruguay es positivo y significativo (efecto apalancamiento), lo que demuestra la presencia de efectos no simétricos de la inflación en la incertidumbre inflacionaria, esto es, que los choques ascendentes presentan un mayor efecto sobre la incertidumbre inflacionaria. Para comprobar la asimetría se estimó la prueba de Engle y $\mathrm{Ng}$ (1993:1757) que permite identificar el sesgo en la magnitud. Los resultados indican que en las economías de Colombia, México, Perú, Tailandia, Filipinas e Indonesia se presenta un mayor impacto en los coeficientes positivos, comparado con los negativos; además de presentar significancia estadística (anexo 1). Este efecto funciona en sentido inverso al apalancamiento, es decir, indica que los cambios en los precios tienden a correlacionarse positivamente con los cambios en la incertidumbre inflacionaria. Dicho de otra manera, esto significa que un choque positivo, las malas noticias, incrementa aún más la incertidumbre inflacionaria, si se les compara con las buenas noticias, representadas por los choques negativos.

Los coeficientes que miden el impacto de la aplicación del régimen de $\mathrm{Ml}$ y su incidencia sobre la incertidumbre inflacionaria muestran que los estimadores para la intersección y la pendiente $\theta_{1}$ y $\theta_{2}$, tanto en su efecto combinado como en su efecto individual, presentan signos negativos y estadísticamente significativos para todas las economías de ambas muestras. Lo anterior, se constituye como evidencia en favor de que la incertidumbre inflacionaria ha disminuido desde 
la implementación de dicho régimen, sobresaliendo el caso de Perú y Filipinas con los coeficientes más elevados. Finalmente, se destaca que la ecuación de la incertidumbre inflacionaria también incluyo los quiebres estructurales identificados en las pruebas de estacionariedad, lo que permitió un mejor ajuste. Resulta interesante que, en el caso de la ecuación de la varianza, para las economías del Sudeste Asiático las variables binarias que capturan los quiebres estructurales son estadísticamente significativas, mientras que en el caso de América Latina estas no presentaron significancia estadística.

\section{Conclusiones}

En la presente investigación se ha estimado la relación de retroalimentación entre el nivel y la volatilidad (incertidumbre) de la inflación para dos de las regiones en desarrollo más importantes: la región latinoamericana (Colombia, México, Perú y Uruguay) y la región del Sudeste Asiático (Tailandia, Corea del Sur, Filipinas e Indonesia). El periodo de análisis es de enero de 1960 a enero de 2018, la razón de que los datos sean mensuales es para capturar el comportamiento estacional que presenta la tasa de cambio porcentual del índice de precios al consumidor.

Se desarrolló un modelo $\operatorname{SARIMA}(p, d, q)(P, D, Q)_{s}-G J R G A R C H(p, q)-M$ para estimar el efecto de retroalimentación entre la media condicional y su varianza condicional; en donde la primera representa el valor esperado de la inflación, en tanto que la segunda mide la incertidumbre inflacionaria. Adicionalmente, la estructura del modelo permite capturar la asimetría detectada que generan los choques positivos y negativos de la incertidumbre inflacionaria sobre la tasa de cambio porcentual de los precios, también se incluye un conjunto de variables de intervención sobre la varianza condicional, que capturan el efecto que ha tenido la implementación del régimen de $\mathrm{Ml}$ en ambas regiones, así como sus quiebres estructurales.

Los resultados indican que: 1) Respecto a la ecuación de la media condicional se identificó que el efecto de la varianza condicional de la inflación (incertidumbre inflacionaria) es positivo y estadísticamente significativo en siete de las ocho economías analizadas (México, Perú, Uruguay, Corea del Sur, Filipinas, Indonesia y Tailandia), evidenciando el cumplimiento de la hipótesis de Cukierman y Meltzer, lo que refleja una posición oportunista de estos institutos centrales; 2) Para la ecuación de la varianza condicional la evidencia presenta resultados mixtos, para el efecto de la inflación rezagada un periodo, que capta su efecto marginal mediante el coeficiente (), se identifica un valor positivo y significativo que sustenta la hipótesis de Friedman y Ball para Colombia, México, Perú, Uruguay, Filipinas y Tailandia; mientras que, el coeficiente se presenta negativo y estadísticamente significativo para Corea del Sur, demostrando así el cumplimiento de la hipótesis de Pourgerami - Maskus; 3) La estructura de los modelos permitió capturar que existe un efecto asimétrico de los choques positivos sobre la tasa de cambio porcentual del índice de precios al consumidor para Colombia, México, Perú e Indonesia, lo que refleja que las malas noticias tienen un mayor impacto que las buenas noticias, sin embargo, en el caso de Uruguay el efecto es inverso; 4) A partir de la adopción del esquema de MI la incertidumbre inflacionaria se ha visto amortiguada, es decir, la volatilidad de la inflación se ha reducido en todas las economías de las dos regiones consideradas. 
La evidencia presentada señala que la persistencia de la volatilidad ha disminuido a partir de la implementación del régimen de $\mathrm{Ml}$; no obstante, los resultados para la región latinoamericana no han sido los esperados en cuanto a crecimiento económico, disminución del desempleo y aumento de inversión en capital. Esto nos habla de una necesidad de políticas monetarias alternativas que sean capaces de lograr mejores resultados en las variables reales cruciales. En este campo, las economías asiáticas han demostrado tener una batería de políticas adecuadas y exitosas que acompañan al régimen de política monetaria, impuesto por el Nuevo Consenso Macroeconómico.

\section{Referencias}

Baillie, R., C.F. Chung y M. Tieslau. 1996. "Analyzing inflation by the fractionally integrated ARFI MA-GARCH model", Journal of Applied Econometrics, 11(1): 23-40.

Ball, L. 1992. "Why does higher inflation raise inflation uncertainty?", Journal of Monetary Eco nomics, 29(3): 371-378. DOI <https://doi.org/10.1016/0304-3932(92)90032-W>.

Batini N. y D. Laxton. 2007. "Under what conditions can inflation targeting be adopted?, The ex perience of emerging markets", en F.S. Mishkin y K. Schmidt-Hebbel (eds) Monetary policy under inflation targeting, Central Bank of Chile, Chile. Recuperado de: http://citeseerx.ist.psu.edu/viewdoc/download?doi=10.1.1.581.7614\&rep=rep1\&type=pdf

Berndt, E., B.H. Hall, R.E. Hall y J.A. Hausman. 1974. "Estimation and inference in nonlinear struc tural models", Annals of Economic and Social Measurement, 3(4): 653-665. Recuperado de: https://www.nber.org/chapters/c10206.pdf.

Bollerslev, T. 1986. "Generalized autoregressive conditional heteroskedasticity", Journal of Econo metrics, vol. 31(3): 307-327. DOI: https://doi.org/10.1016/0304-4076(86)90063-1.

Bredin, D., J. Elder y S. Fountas. 2009. "Macroeconomic uncertainty and performance in Asian countries", Review of Development Economics, 13(2): 215-229. DOI: https://doi.org/10.1111/ j.1467-9361.2009.00508.x

Brito, R.D. y B. Bystedt. 2010. "Inflation targeting in emerging economies: panel evidence", Journal of Development Economics, 91(2010): 198-210, Elsevier. Recuperado de: https://faculty.smu. edu/millimet/classes/eco6375/papers/brito\%20bystedt\%202010.pdf

Broto, C. 2008. "Inflation targeting in Latin America: empirical analysis using GARCH models." Do cumentos de Trabajo Banco de España, núm. 0826. Recuperado de: https://www.bde. es/f/webbde/SES/Secciones/Publicaciones/PublicacionesSeriadas/DocumentosTrabajo/08/ Fic/dt0826e.pdf

Caballero C.B. y R. Caballero. 2015. "Efectos asimétricos de shocks de inflación e incertidumbre inflacionaria en Bolivia 1990-2013". Economía Informa, 394: 68-99. DOI: https://doi.org/10.1016/i.ecin.2015.09.006

Calvo, G.A. y F.S. Mishkin, 2003. "The mirage of exchange rate regimes for emerging market coun tries", Journal of economic Perspective 17(4): 99-118. Recuperado de: https://pdfs.semantics cholar.org/038b/aa10db6b56aeeffe272fed5b919018e1d17a.pdf 
Campbell, A. 1987. "Stock returns and term structure", Journal of Financial Economics, 18(2): 373399. DOI: https://doi.org/10.1016/0304-405X(87)90045-6

Capistrán, C. y M. Ramos-Francia. 2009. "Inflation dynamics in Latin America”, Contemporary Economic Policy, Western Economic Association International, 27(3): 349-362. DOI: https://doi.org/10.1111/i.1465-7287.2008.00128.x

Carrasco, C.A. y J. Ferreiro. 2011, "Inflation targeting and economic performance: the case of Mexico", Panoeconomicus, special issue, LVIII (5): 675-692. Recuperado de: https://scindeksclanci.ceon.rs/data/pdf/1452-595X/2011/1452-595X1105675C.pdf

Castillo, L. y J. Ramoni-Perazzi. 2017. La volatilidad del tipo de cambio paralelo en Venezuela 2005-2015, Apuntes del Cenes, 36(63): 95-235. DOI: http://dx.doi.org/10.19053/01203053. v36.n63.2017.5312

Chen S.W., C. H. Shen y Z. Xie. 2006. "Nonlinear relationship between inflation and infla tion uncertainty in Taiwan", Applied Economics Letters, 13(8): 529-533. DOI: https://doi. org/10.1080/13504850500400405

Cukierman, A. y A. Meltze. 1986. "A theory of ambiguity, credibility, and inflation under discre tion and asymmetric information", Econometrica, 54(5): 1099-1128. Recuperado de https:// pdfs.semanticscholar.org/27ee/6dfa9bdb8c102c9e2839e030f56d79e0c13a.pdf

Daal, E., A. Naka y B. Sanchez. 2005. "Re-examining inflation and inflation uncertainty in devel oped and emerging countries", Economics Letters, 89(2): 180-186. Recuperado de: https:// pdfs.semanticscholar.org/fde5/f88a4cc60314b506b58b787d1d2e581cec82.pdf

Enders, W. 2015. Applied Econometric Time Series, New York, John Wiley.

Engle, R., D. Lilien y R. Robins. 1987. "Estimating time varying risk premia in the term structure: The Arch-M model", Econometrica, 55(2): 391-407. Recuperado de: https://www.jstor.org/ stable/1913242

Engle, R.F. y V.K. Ng. 1993. "Measuring and testing the impact of news on volatility, Journal of Finance, 48(5): 1749-1778. DOI: https://doi.org/10.1111/j.1540-6261.1993.tb05127.x

Engle, R.F. 1982. "Autoregressive conditional heteroskedasticity with estimates of the variance of United Kingdom inflation", Econometrica, 50(4): 987-1007. Recuperado de: https://absalon. ku.dk/courses/21267/files/1645798/download?

Epstein, G. y E. Yeldan. 2008. "Inflation targeting, employment creation and economic develop ment: assessing the impacts and policy alternatives", International Review of Applied Eco nomics, 22(2): 131-144. DOI: https://doi.org/10.1080/02692170701880601.

Evans, M. 1991. "Discovering the link between inflation rates and inflation uncertainty", Jour nal of Money, Credit, and Banking, 23(2): 169-184. Recuperado de: https://www.jstor.org/ stable/1992775

Fama, M. 1963. "Risk returm and equilibrium: empirical test", Journal of Political Economy, 81(3): 607-636.

Figlewski, S. 1997. "Forecasting volatility", Financial Markets, Institutions and Instruments, 6(1): 1-88. DOI: < https://doi.org/10.1111/1468-0416.00009>.

Fountas, S. 2010. "Inflation, inflation uncertainty and growth: are they related?", Economic Modelling, 27(5): 896-899. DOI: https://doi.org/10.1016/i.econmod.2010.06.001. 
Frankel, J.A. 2010. Monetary policy in emerging markets: a survey, NBER Working Paper Series, núm. 16125. Recuperado de https://www.nber.org/papers/w16125.pdf

Friedman, M. 1977. "Nobel lecture: inflation and unemployment", Journal of Political Economy, 85(3): 451-472. Recuperado de: https://edisciplinas.usp.br/pluginfile.php/1130235/mod re source/content/1/friedman\%20milton\%20\%28nobel\%20lecture\%20inflation\%20and\%20 unemployment\%29.pdf

Glosten, L., R. Jagannathan y D. Runkle. 1993. “On the relation between the expected value and the volatility of the normal excess return on stocks", The Journal of Finance, 48(5): 17791801. Recuperado de https://faculty.washington.edu/ezivot/econ589/GJRJOF1993.pdf.

Golob, J. 1994. "Does inflation uncertainty increase with inflation?", Federal Reserve Bank of Kansas City Economic Review, núm. 3. Recuperado de: https://www.kansascityfed.org/pu blicat/econrev/pdf/3q94golb.pdf

Gonçalves, C.E. y J. Salles. 2008. "Inflation targeting in emerging economies: what do the data say?" Journal of Development Economics, 85(1-2): 312-318. DOI: https://doi.org/10.1016/j. jdeveco.2006.07.002

Grier, K.B. y M. Perry. 2000. "The effects of real and nominal uncertainty on inflation and output growth: some GARCH-M evidence", Journal of Applied Econometrics, 15(1): 45-58.

Grier, R. y K.B. Grier. 2006. "On the real effects of inflation and inflation uncertainty in México", Journal of Development Economics, 80(2): 478-500. DOI: https://doi.org/10.1016/i.jde veco.2005.02.002

Hentschel, L. 1995. "All in the family nesting symmetric and asymmetric GARCH models", Journal of Financial Economics, 39: 71-104. Recuperado de: http://citeseerx.ist.psu.edu/viewdoc/do wnload?doi=10.1.1.557.8941\&rep=rep1\&type $=$ pdf

Holland, A.S. 1995. "Inflation and uncertainty: tests for temporal ordering", Journal of Money, Credit and Banking, 27(3): 827-837. Recuperado de: https://www.jstor.org/stable/2077753.

Holland, A.S. 1993. "Uncertain effects of money and the link between the inflation rate and infla tion uncertainty", Economic Inquiry, 31(1): 39-51. DOI: https://doi.org/10.1111/j.1465-7295. 1993.tb00864.x

Hyndman, R.J. y G. Athanasopoulos. 2018. Forecasting: Principles and Practice, OTexts. Recupera do de: https://www.otexts.org/fpp.

Jiranyakul, K. y T.P. Opiela. 2010. "Inflation and inflation uncertainty in the ASEAN-5 economies", Journal of Asian Economics, 21(2):105-112. DOI https://doi.org/10.1016/ j.asieco.2009.09.007

Kontonikas, A. 2004. "Inflation and inflation uncertainty in the United Kingdom, evidence from GARCH modelling", Economic Modelling 21(3): 525-543. DOI https://doi.org/10.1016/i. econmod.2003.08.001

Lim, J. 2008. "Central banking in the Philippines: from inflation targeting to financing development," International Review of Applied Economics, 22(2): 271-285. DOI: https://doi. org/10.1080/02692170701880791

Lin, S. y H. Ye. 2009. "Does inflation targeting make a difference in developing countries?", Journal of Development Economics, 89(1): 118-123. DOI: https://doi.org/10.1016/i.jdeveco.2008.04.006 
López, H.F. 2004. "Modelado de la volatilidad y pronóstico del Índice de Precios y Cotizaciones de la Bolsa Mexicana de Valores", Contaduría y Administración. 213: 43-72. Recuperado de: http://www.redalyc.org/articulo.oa?id=39521303.

Mandelbrot, B. 1963. "The variation of certain speculative prices", Journal of Business, 36(4): 394419. Recuperado de: https://web.williams.edu/Mathematics/sjmiller/public html/341Fa09/ econ/Mandelbroit VariationCertainSpeculativePrices.pdf

Makridakis, S., S.C. Wheelwright y R.J. Hyndman. 1998. Forecasting: methods and applications, New York: John Wiley \& Sons.

McDermott, J. y P. McMenamin. 2008. Assessing inflation targeting in Latin America with a DSGE model, Central Bank of Chile Working Papers, núm. 469. Recuperado de: https://dialnet. unirioja.es/descarga/articulo/2868654.pdf

McLeod, A. y W. Li. 1983. "Diagnostic checking ARMA time series models using squared residual autocorrelations", Journal of Time Series Analysis, 4(4): 269-273. Recuperado de: https:// onlinelibrary.wiley.com/doi/abs/10.1111/j.1467-9892.1983.tb00373.x

Miles, W. 2009. "Central Bank independence, inflation and uncertainty: the case of Colombia", In ternational Economic Journal, 23(1): 65-79. DOI:https://doi.org/10.1080/10168730802696624

Mishkin, F. 2000. "Inflation targeting in emerging-market countries", The American Economic Re view, 90(2): 105-109. Recuperado de: http://www.jstor.org/stable/117201

Mishkin, F.S. 2006. Monetary policy strategy: how did we get here? NBER Working Papers Series, núm. 12515. Recuperado de: https://ideas.repec.org/p/nbr/nberwo/12515.html

Mishkin, F.S. 2008. Does stabilizing inflation contribute to stabilizing economic activity? NBER Working Paper Series, núm. w13970. Recuperado de: https://papers.ssrn.com/sol3/papers. cfm?abstract id $=1131623$

Mohd, S. H., Baharumshah, A. Z., \& Fountas, S. (2013). Inflation, inflation uncertainty and output growth: Recent evidence from ASEAN-5 countries. Singapore Economic Review, 58(4). Recu perado de: <https://doi.org/10.1142/S0217590813500306>

Morón, E. y D. Winkelried.2005. “Monetary policy rules for financially vulnerable economies", Jour nal of Development Economics, 76(1): 23-51. DOI: https://doi.org/10.1016/i.jdeve co.2004.08.001

Nelson, D.B. 1991. "Conditional heteroskedasticity in asset returns: a new approach", Econometrica, 59(2): 347-370. Recuperado de: https://klevas.mif.vu.lt/ danas/TS/ged.pdf.

Perrotini H.I. y B.D. Rodríguez. 2012. "Inflación, incertidumbre inflacionaria y crecimiento econó mico en México: 1929-2009", Revista Mexicana de Economía y Finanzas, 7(1): 1-26. DOI: https://doi.org/10.21919/remef.v7i1.23

Posedel, P. 2005. "Properties and estimation of GARCH (1,1) model", Metodolski zvezki, 2(2): 243257. Recuperado de: https://www.stat-d.si/mz/mz2.1/posedel.pdf

Posen, A. 2008. "The Future of inflation targeting", Challenge 51(4): 5-22. DOI: https://doi. org/10.2753/0577-5132510401

Pourgerami, A. y K. Maskus.1987. "The effects of inflation on the predictability of price changes in Latin America: some estimates and policy implications", World Development 15(2): 287-290. DOI https://doi.org/10.1016/0305-750X(87)90083-0 
Roger, S. (2009) "Inflation Targeting at 20: Achievements and Challenges". IMF Working Paper 09/236. Disponible en: https://ssrn.com/abstract=1497235

Rosas R.E. y T. López G. 2018. "Inflación e incertidumbre inflacionaria: la postura del Banco de México, 1969-2017", Finanzas y Política Económica, 10(2): 349-372. Recuperado de: http:// dx.doi.org/10.14718/revfinanzpolitecon.2018.10.2.6

Rosas R.E. y D.M. Mimbrera. 2018. "Inflación y volatilidad cambiaria en México (1969-2017)". En sayos de Economía, 28(53): 37-64. Recuperado de https://revistas.unal.edu.co/index.php/ ede/article/view/75057/69783

Ungar, M. y B. Zilberfarb. 1993. "Inflation and its unpredictability -theory and empirical evi dence", Journal of Money, Credit, and Banking, 25(4): 709-720. Recuperado de: https://www. jstor.org/stable/2077800

Vega, M. y D. Winkelried. 2005. "Inflation targeting and inflation behavior: a successful story?", International Journal of Central Banking, 1(3): 153-175. Recuperado de: https://www.ijcb.org/ journal/ijcb05q4a5.pdf

Vogelsang, T. J. y P. Perron. 1998. "Additional tests for a unit root allowing for a break in the trend at an unknown time", International Economic Review, 39(4): 1073-1100.

Walsh, C.E. 2009. "Inflation targeting: what have we learned?", International Finance, 12(2): 195233. Recuperado de: https://doi.org/10.1111/i.1468-2362.2009.01236.x.

Wilson, B.K. 2006. "The links between inflation, inflation uncertainty and output growth: new time series evidence from Japan", Journal of Macroeconomics, 28(3): 609-620. DOI: https:// doi.org/10.1016/j.jmacro.2004.11.004

Zakoïan, J.M. 1994. "Threshold heteroskedastic models", Journal of Economic Dynamics and Control, 18(5): 931-955. DOI: https://doi.org/10.1016/0165-1889(94)90039-6

Anexo 1. Prueba de magnitud de Engle y $\mathrm{Ng}$ 


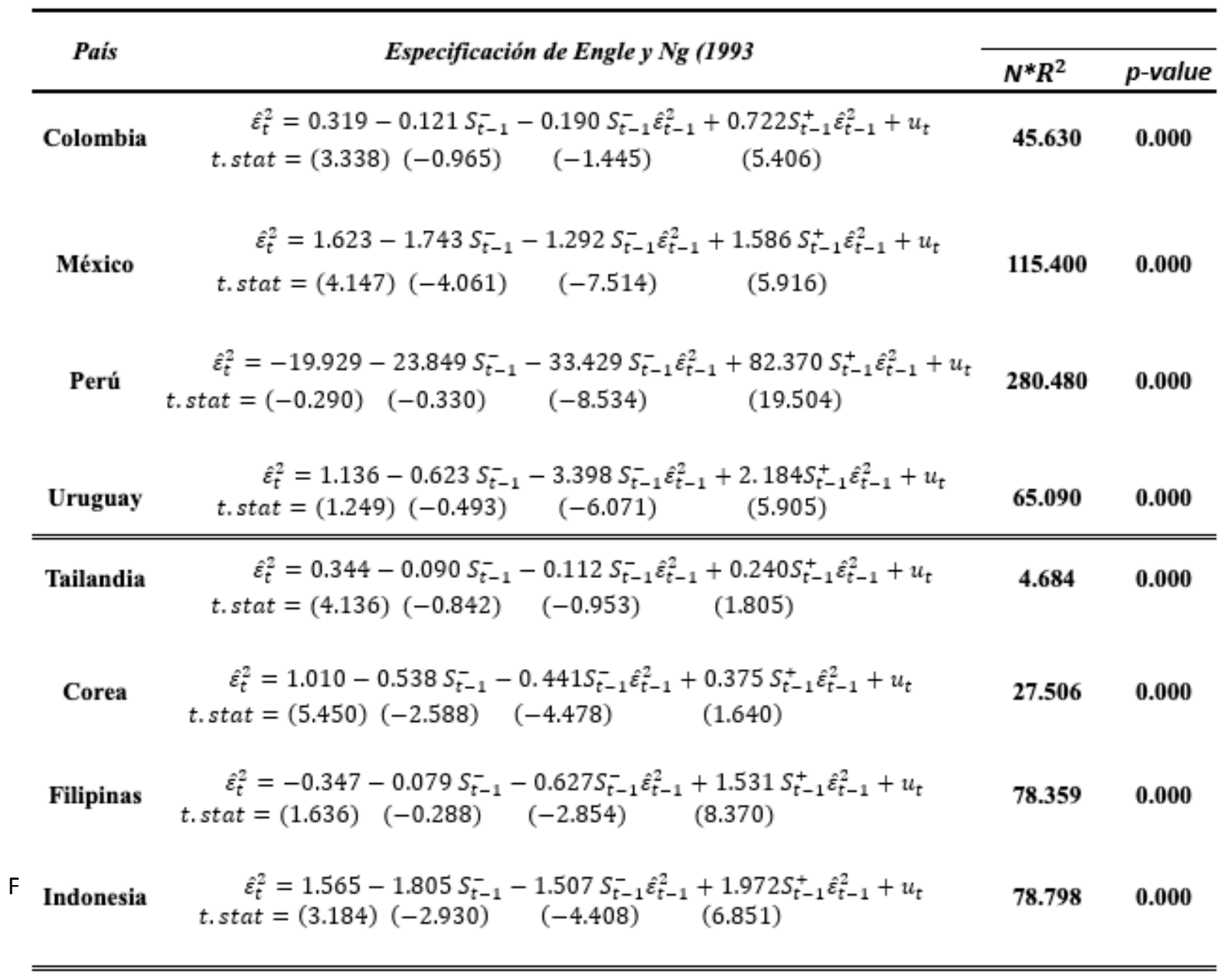

